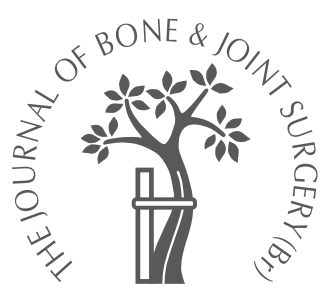

R. K. Kadiyala,

A. Ramirez,

A. E. Taylor,

C. L. Saltzman,

M. D. Cassell

From The University

of Iowa, Iowa City,

USA

R. K. Kadiyala, MD, PhD,

Assistant Professor of

Orthopaedic Surgery

Mt. Sinai Medical Center,

MSOP Suite 690, 4302 Alton

Road, Miami Beach, Florida

33140, USA.

A. Ramirez, MD, Staff

Surgeon

7205 Clarendon Road,

Bethesda, Maryland 20814-

2319, USA.

A. E. Taylor, MPH,

Orthopaedic Surgery

Research Assistant

C. L. Saltzman, MD

Professor of Orthopaedic

Surgery and Engineering

M. D. Cassell, PhD

Professor of Anatomy and

Cell Biology

University of lowa Hospitals

and Clinics, 200 Hawkins

Drive, lowa City, lowa 52242,

USA.

Correspondence should be sent to Professor M. D.

Cassell; e-mail:

martin-cassell@uiowa.edu

C2005 British Editorial

Society of Bone and

Joint Surgery

doi:10.1302/0301-620X.87B3.

$15694 \$ 2.00$

$J$ Bone Joint Surg [Br]

2005;87-B:337-42.

Received 3 June 2004;

Accepted after revision

7 September 2004

\title{
The blood supply of the common peroneal nerve in the popliteal fossa
}

We investigated the blood supply of the common peroneal nerve. Dissection of 19 lower
limbs, including six with intra-vascular injection of latex, allowed gross and microscopic
measurements to be made of the blood supply of the common peroneal nerve in the
popliteal fossa. This showed that a long segment of the nerve in the vicinity of the fibular
neck contained only a few intraneural vessels of fine calibre. By contrast, the tibial nerve
received an abundant supply from a constant series of vessels arising directly from the
popliteal and posterior tibial arteries.
The susceptibility of the common peroneal nerve to injury from a variety of causes and its lack of response to operative treatment may be explained by the tenuous nature of its intrinsic blood supply.

Palsy of the common peroneal nerve is a frequent complication of various orthopaedic conditions, ${ }^{1-7}$ as well as of orthopaedic and other surgical procedures. ${ }^{8-15}$ Direct injuries, ${ }^{7,11}$ and compression, torsion or stretching of the nerve $e^{1,3,8,15}$ are thought to be the cause of these lesions, but embolic and thrombotic events have also been implicated. Palsy of the common peroneal nerve is a recognised complication of intra-aortic balloon pumping ${ }^{16-18}$ and isolated cases have been reported after iliac endarterectomy, aortoiliac bypass and femoral aortography. ${ }^{8}$ Ischaemia of nerves in the calf has been implicated as the cause of palsy of the common peroneal nerve, ${ }^{19}$ and Ferguson and Liversedge $^{20}$ reported seven cases of foot drop which were associated with a local vascular abnormality. In another series of 145 patients with vascular claudication, $54(37 \%)$ had some neurological impairment primarily involving the peroneal nerve and eight presented with complete paralysis of muscles innervated by the common peroneal nerve. ${ }^{21}$ These observations strongly suggest that the blood supply of the common peroneal nerve may be vulnerable to a variety of traumatic and pathological insults. ${ }^{22}$ Furthermore, it may lack sufficient collateral connections to avoid ischaemia.

The gross blood supply of the sciatic nerve and its divisions has been well described. ${ }^{22,23}$ The common peroneal nerve appears to receive its blood supply directly from muscular, cutaneous and geniculate branches of the popliteal artery in the popliteal fossa. This and other peripheral nerve axons are susceptible to ischaemia because of the length of their axons from the cell bodies. However, some protection from ischaemic neuropathy is conferred by the extent of the anastomoses between the many vessels. $^{24}$ Lundborg and Brånemark ${ }^{25}$ first observed the nature of these vessels, describing a many-layered overlap so that there is no single essential artery.

Despite Sunderland's review of earlier reports and his own extensive studies, ${ }^{22,23}$ many details concerning the number and extent of intraneural vessels (vasa nervorum) arising from the circumflex fibular artery as it accompanies the common peroneal nerve around the neck of the fibula are lacking. Flanigan, Cassell and Saltzman ${ }^{26}$ described several new anatomical features, including poor anastomoses and asymmetrical branching of the vasa nervorum, which may predispose the tibial nerve to neuropathy. Since mononeuropathies of the common peroneal nerve are more common than those of the tibial nerve, deficiencies in intraand extraneural vascular anastomoses may play a major and heretofore unrecognised role in this distinction. We have examined in detail the origin of the blood supply to the common peroneal nerve in the popliteal fossa and around the neck of the fibula in order to determine the organisation of the intraneural supply.

\section{Materials and Methods}

All specimens used in our study were obtained from donors to the University of Iowa Deeded 


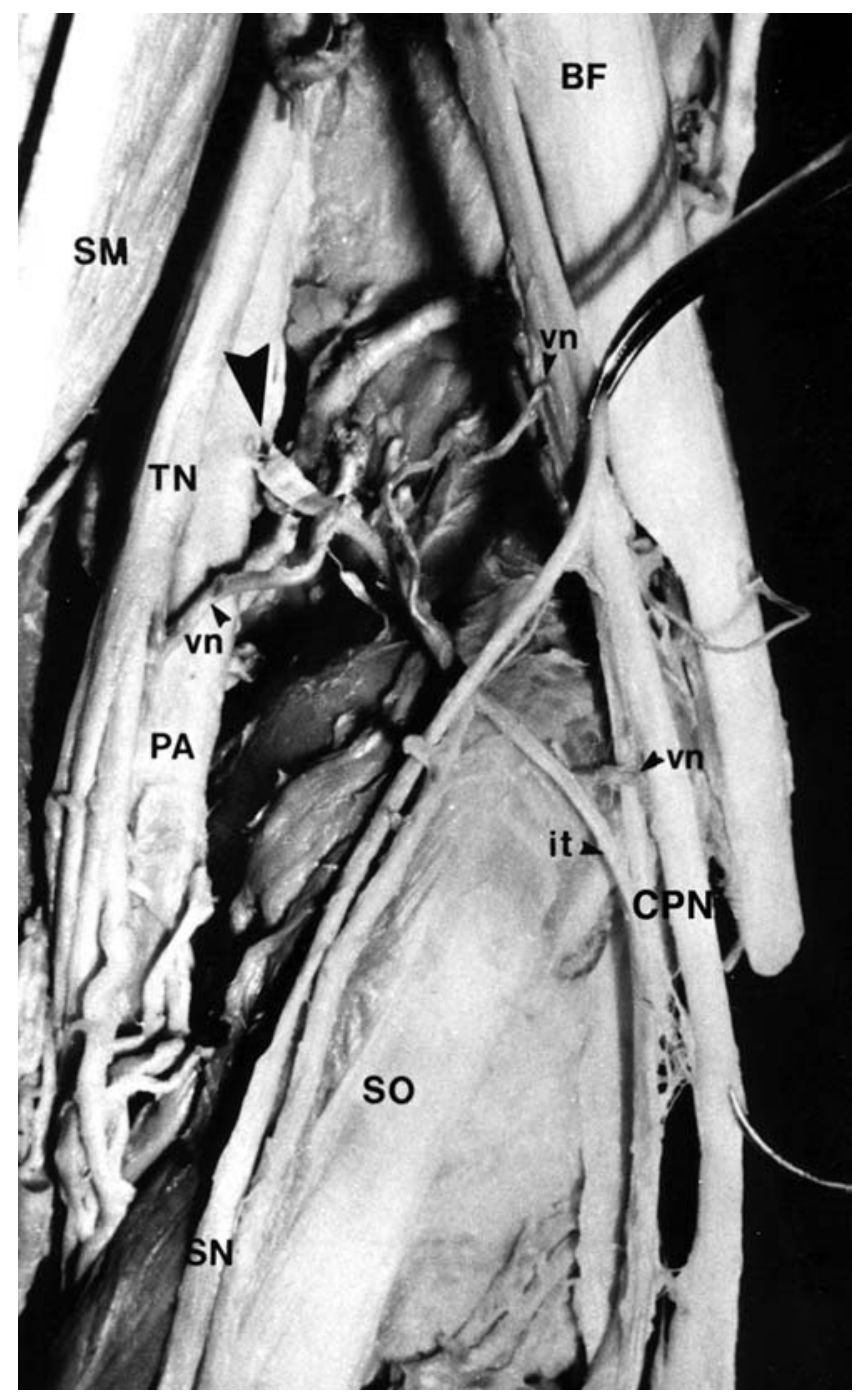

Fig. 1

Photograph of the gross dissection of the popliteal fossa of the right leg. The vessel (arrow) branching from the popliteal artery (PA) gives rise to vasa nervorum ( $\mathrm{vn}$ ) to the tibial nerve (TN) and common peroneal nerve (CPN) and a branch which bifurcates into a vessel accompanying the sural nerve (SN) and the epineurial vessel (it) running with the common peroneal nerve (BF, biceps femoris; SM, semimembranosus; SO, soleus).

Body Program and were embalmed using carotid arterial perfusion of $2 \%$ formaldehyde containing $0.5 \%$ phenol and $5 \%$ ethanol. Six embalmed lower limbs from four subjects were perfused with saline through an infusion pump by way of the femoral artery in order to remove any remaining clots and debris. Perfusion was continued until clear fluid was observed passing out through the femoral vein. Approximately $500 \mathrm{ml}$ of red or blue coloured latex (Ward's Natural Science, Rochester, New York) were then perfused through the femoral artery at moderate pressure over three to five minutes. It was allowed to harden for 24 to 48 hours after which the specimens were dissected. This usually involved removal of the skin from the lower thigh to the ankle followed by careful blunt dissection of the popliteal fossa and its contents, and mobilisation of the common peroneal and tibial nerves from the bifurcation of the sciatic nerve to the middle of the lateral and posterior crural compartments, respectively. The blood supply of the common peroneal nerve and tibial nerve was photographed and recorded, after which both, together with the popliteal artery and its branches, was removed in their entirety, dehydrated in increasing concentrations of ethanol and cleared in three changes of methylsalicylate. The cleared specimens were subsequently photographed under transillumination.

In addition, the popliteal fossae in another 13 lower limbs, without vascular injections of latex, were dissected and the blood supply of the common peroneal nerve investigated.

\section{Results}

Of the six latex-injected specimens, five showed a similar arrangement of the vasa nervorum supplying the common peroneal nerve in the popliteal fossa. A single artery about $1 \mathrm{~mm}$ in diameter branched directly from the popliteal artery at approximately the mid-point of the popliteal fossa (Fig. 1). This vessel coursed laterally to give a large branch to the common peroneal nerve just distal to the bifurcation of the sciatic nerve. In the case illustrated in Figure 1, it also gave a small branch to the tibial nerve. After giving off the initial vessel to the common peroneal nerve, it branched into a larger vessel which accompanied the sural communicating branch of the common peroneal nerve, and a smaller vessel which entered the fibrous sheath of the common peroneal nerve (Figs 1 and 2a). After running with the common peroneal nerve for 30 to $60 \mathrm{~mm}$, this epineurial vessel divided into a large branch forming a neurovascular bundle with the lateral sural nerve, and a smaller vessel which continued with the common peroneal nerve, eventually anastomosing with a superficial branch of the anterior recurrent tibial artery. In the five specimens containing this arrangement, the mean distance between the large proximal branch entering the common peroneal nerve and the anterior recurrent tibial artery was $116 \mathrm{~mm}$. The mean distance between the vasa nervorum entering the tibial nerve directly from the popliteal and the posterior tibial arteries was $41 \mathrm{~mm}$.

In the sixth remaining injected specimen, the proximal part of the blood supply of the common peroneal nerve had a slightly different arrangement (Fig. 2b). A single vessel arose from the mid-point of the popliteal artery and immediately gave off a small branch to the common peroneal nerve at a point from which the lateral sural nerve branched. This artery then passed anterior to the popliteal artery and gave a large vessel to accompany the sural nerve, before passing medially to enter the distal aspect of semimembranosus. The small vessel entering the proximal part of the common peroneal nerve gave off a small branch which ran within the sheath of the common peroneal nerve, anastomosing distally with a branch of the anterior recurrent tibial artery. 


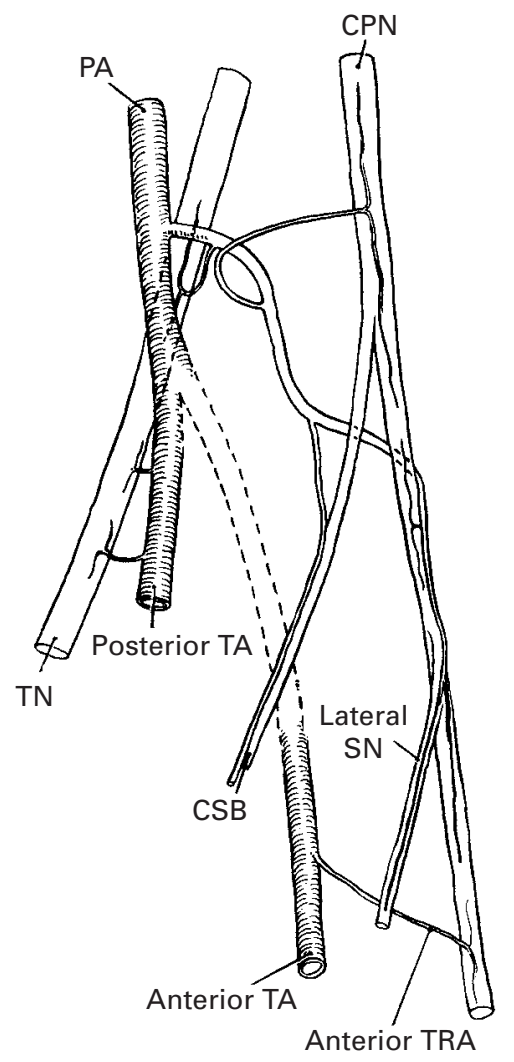

Fig. 2a

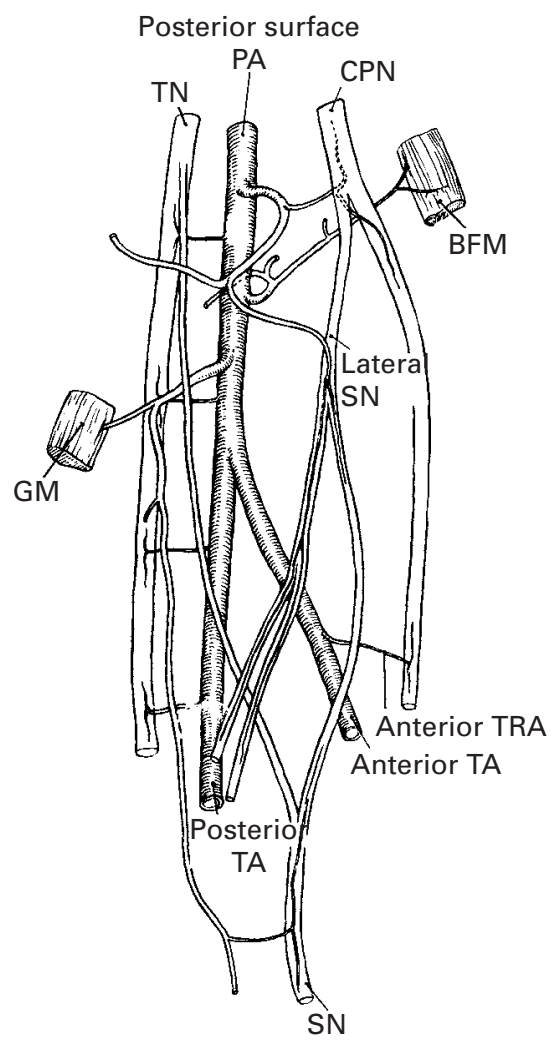

Fig. 2b

Diagrams of the major vascular arrangements supplying the CPN in the popliteal fossa showing a) the arrangement illustrated in Figure 1 found in 11 cases (5/6 injected; 6/13 non-injected) and b) that found in one injected specimen (BFM, biceps femoris muscle; CPN, common peroneal nerve; CSB, communicating sural bridge; GM, gastrocnemius muscle; PA, popliteal artery; $\mathrm{SN}$, sural nerve; TA, tibial artery; TN, tibial nerve; TRA, tibial recurrent artery).

In the 13 non-injected specimens, six were shown to have a pattern of supply to the common peroneal nerve similar to that of the five injected specimens, although none had the arrangement found in the sixth injected specimen. In the remaining seven non-injected specimens, it was not possible to demonstrate epineurial vessels or vasa nervorum derived from a branch of the popliteal artery. In four of these specimens, a large muscular branch of the popliteal artery, supplying biceps femoris and adductor magnus, gave a vessel which entered the common peroneal nerve close to its origin from the sciatic nerve. In one of these specimens, the sciatic nerve divided high, in the mid-thigh. However, no other vasa nervorum were seen distal to this in these specimens, suggesting that vessels may have been damaged during the dissection of the popliteal fossa. Similarly, in the remaining three specimens, no vasa nervorum arising from the popliteal artery were observed although longitudinal anastomotic channels were seen within the common peroneal nerve.

Histological examination. In the four cleared specimens, latex-filled vessels as small as $50 \mu \mathrm{m}$ in diameter were observed (Fig. 3). This diameter corresponds to the mean diameter of the smallest vessels (capillaries) found in previous studies, ${ }^{27,28}$ suggesting that the vessels of the smallest calibre were filled with latex. In three specimens, a striking paucity of apparent intraneural vessels was observed in the proximal part of the common peroneal nerve. In these specimens, which were all supplied proximally by way of a direct branch of the popliteal artery, the first vasa nervorum derived from the popliteal branch entered the nerve and bifurcated symmetrically into two longitudinal vessels running proximally and distally. These vessels, located in the epineurium, gave rise to a number of small vessels of fine calibre, which extended for 20 or $30 \mathrm{~mm}$ within the substance of the common peroneal nerve. The next vessel to enter the common peroneal nerve branched from the proximal part of the long anastomotic vessel connecting the popliteal branch with the anterior recurrent tibial artery. This vessel bifurcated on entering the nerve, but in every case the ascending ramus was considerably larger than the descending ramus. It formed an anastomosis with the descending branch of the previous vessel whereas the thin descending ramus gave off one or two extremely fine vessels which could be followed within the substance of the nerve 


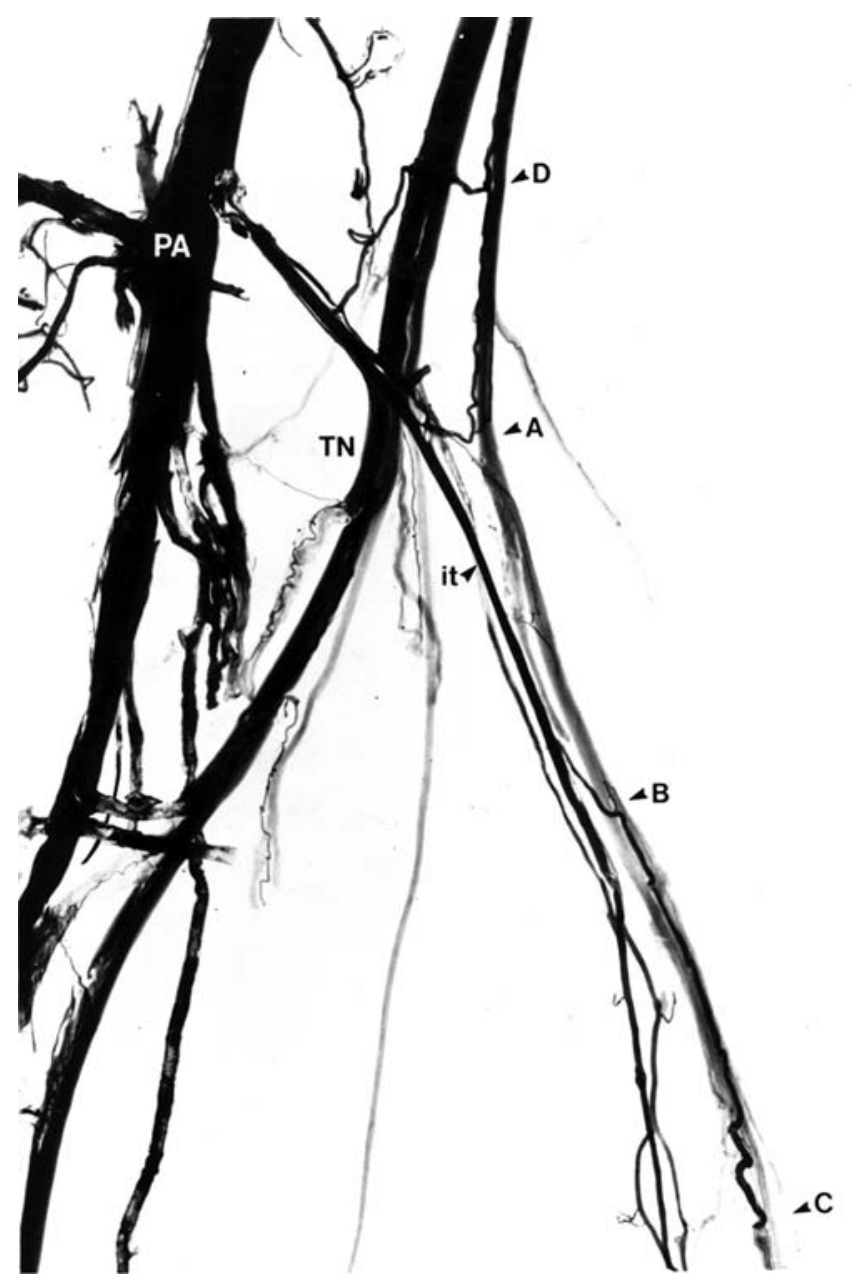

Fig. 3

Transilluminated view of the intraneural supply of the common peroneal nerve in the popliteal fossa in a cleared specimen. There is asymmetrical branching of the vasa nervorum entering the common peroneal nerve at $A$ and $B$ and a paucity of intraneural vessels between these two points. A symmetrically branching vasa nervorum derived from a branch of the popliteal artery can be seen at $C$. The connection with the anterior recurrent tibial artery occurred at $D$ but was severed when the nerve bundle specimen was removed from the leg (PA, popliteal artery; TN, tibial nerve; it, epineurial vessel).

for about 10 to $30 \mathrm{~mm}$. The next tributary, from the long anastomotic vessel, entered the common peroneal nerve at approximately the level of the head of the fibula. This vessel also bifurcated asymmetrically and its larger descending ramus gave off numerous vessels of fine calibre into the common peroneal nerve and joined the anterior recurrent tibial artery. No anastomoses between the fine descending and ascending vessels derived from the two vasa nervorum were seen, although in the specimen illustrated (Fig. 3) a vessel of fine calibre entered the common peroneal nerve at approximately midway between these two vasa nervorum. In all three specimens, the common peroneal nerve segment, with a few intraneural vessels, extended from the edge of the tendon of biceps femoris to the neck of the fibula.

\section{Discussion}

We have investigated the blood supply of the common peroneal nerve in the popliteal fossa. Our four major findings are that: 1) the blood supply to the proximal part of the nerve is derived in approximately two-thirds of cases from a direct branch of the popliteal artery; 2) this branch also provides an anastomotic vessel which runs in the connective-tissue sheath of the nerve and anastomoses with the anterior recurrent tibial artery; 3) vasa nervorum derived from this anastomotic vessel enter the nerve and appear to bifurcate asymmetrically; and 4) this asymmetrical branching results in a long segment of the nerve containing only a few intraneural vessels of fine calibre. Compared with the tibial nerve, which received symmetrically branching vasa nervorum at approximately every $4 \mathrm{~cm}$, from the popliteal and posterior tibial arteries, the blood supply to the common peroneal nerve in the popliteal fossa appeared to be relatively sparse. The apparently poor vascularisation of this segment of the CPN as it approaches the fibular neck may predispose the nerve to ischaemic damage through direct compression or tension as well as embolic and other vascular complications.

The results of the gross analysis appear to be broadly consistent with the previous extensive studies of the blood supply of the common peroneal nerve $\mathrm{e}^{22,29}$ although they suggest that supply directly from a branch of the popliteal artery is the most common arrangement. Sunderland ${ }^{22}$ found that only 12 of 40 nerves examined received one or two vasa nervorum from the popliteal artery directly, and only in three cases was it the exclusive source. This discrepancy may be due to the small sample size in our study. Alternatively, the use of injections of latex may have facilitated identification of popliteal-derived vessels. Additional vasa nervorum from geniculate, muscular and perforating arteries have been reported in nerves both with and without popliteal branches. We were unable to identify any contribution to the common peroneal nerve from the geniculate arteries although contributions from muscular branches were found in four non-injected cases. Tonkoff ${ }^{29}$ described and illustrated the blood supply of the common peroneal nerve from a musculocutaneous branch of the popliteal artery. The direct branch from the popliteal artery, identified here as providing the proximal supply to the nerve in the popliteal fossa, consistently provided cutaneous branches which ran with the sural communicating and lateral sural nerves. However, muscular branches arising from this vessel were only seen in one injected case.

Sunderland ${ }^{22}$ described the vasa nervorum of the common peroneal nerve in the vicinity of the fibular neck as arising from the circumflex fibular artery which, he reported, accompanied the nerve at this point. By contrast, Tonkoff ${ }^{29}$ and $\mathrm{Hyrtl}^{30}$ described the vasculature as arising from the anterior tibial artery; Tonkoff delineated the branch as the ascending nutrient artery of the anterior recurrent tibial artery and Hyrtl delineated it as the fibular branch of the anterior tibial artery. In our material, the 
artery which accompanied the common peroneal nerve around the fibular neck was a branch from the distal continuation of the anastomotic vessel which joined with the anterior tibial recurrent artery. The circumflex fibular artery arose in all cases from the posterior tibial artery and passed closely round the fibular neck to join the recurrent tibial artery deep to tibialis anterior. No vasa nervorum supplying the nerve arose from the circumflex fibular artery, nor were any seen in the cleared specimens. On this evidence, it seems likely that Sunderland ${ }^{22}$ misidentified the vessel accompanying the common peroneal nerve around the fibular neck as the circumflex fibular artery.

One of the more important findings of our study was the observation of asymmetrical branching of the vasa nervorum derived from the anastomotic vessel. In three of the four cleared specimens, the proximal vessel gave rise to a large ascending and a much smaller descending ramus, whereas the distal vessel gave off a small ascending and a large descending ramus. Consequently, only vessels of small calibre supplied a long segment of the common peroneal nerve. On either side of this segment, the large rami anastomosed with those from adjacent vasa nervorum, forming a continuous anastomotic chain of large calibre, from which numerous intraneural vessels arose. Proximally, the anastomotic chain supplying the nerve was continuous with the arteria comitans nervi ischiadici supplying the sciatic nerve, and distally it connected with bifurcating vasa nervorum derived from the anterior tibial artery. The continuous and richly anastomosed vascular supply to the nerve thus appears to be tenuous only in the segment of the common peroneal nerve around the neck of the fibula.

The popliteal fossa is a developmental watershed where several vascular rearrangements occur because of regression and replacement of the embryonic axial artery of the lower limb. Proximal to the tendinous hiatus, at which point the embryonic axial artery receives the developing femoral artery and continues as the popliteal artery, the axial artery is vestigial in the adult and is represented for the most part by the arteria comitans nervi ischiadici. ${ }^{31}$ Hence, the vessels which we observed running from the popliteal artery to the sciatic and common peroneal nerves may represent a surviving portion of the axial artery. However, the anterior tibial artery, a vessel not directly derived embryologically from the axial artery, ${ }^{31,32}$ supplies the distal portion of the common peroneal nerve. Therefore, the asymmetries in the intraneural blood supply of the nerve around the fibular neck may reflect the different embryonic origins of the main arterial trunks supplying proximal and distal portions of it. Clearly, this suggestion requires further study, but it is noteworthy that similar vascular asymmetries in the intraneural supply of the tibial nerve have been observed near the junction of the medial and lateral plantar arteries. ${ }^{28}$ These vessels have distinct embryological origins. ${ }^{31}$

In addition to the many reports of known vascular events leading to palsy of the common peroneal nerve there is sur- gical evidence which suggests the importance of the vascular supply of the nerve around the fibular head. Rose et $\mathrm{al}^{13}$ reviewed cases of palsy of the common peroneal nerve after total knee arthroplasty in 435 knees with flexion contracture. In this group, 35 patients had a prophylactic exploration and release of the nerve. Of these, five $(15 \%)$ developed palsy of the common peroneal nerve after surgery. In the remaining 400 patients with flexion contractures of the knee, only nine $(2 \%)$ developed a palsy. Rose et $\mathrm{al}^{13}$ concluded that vascular compromise and/or stretching of the nerve were significant aetiological factors.

Mont et $\mathrm{al}^{33}$ described the operative findings of epineural fibrosis and bands of fibrous tissue constricting the common peroneal nerve in a series of 31 patients undergoing operative decompression for peroneal palsy. They noted these changes at the level of the fibular head and the proximal origin of the tendon of peroneus longus, an area which corresponds to the region of vascular paucity of the common peroneal nerve noted in our study.

On the basis of these findings, and the anatomical data presented here, surgeons operating around the common peroneal nerve should be aware of its intrinsically delicate blood supply in the vicinity of the fibular head. Further study of vascular compromise and the possible indication for anticoagulation in selected patients presenting with acute peroneal palsy is warranted.

No benefits in any form have been received or will be received from a commercial party related directly or indirectly to the subject of this article.

\section{References}

1. Bowman AJ Jr, Kilfoyle RM, Broom JS. Varus injury of the knee with common peroneal nerve palsy. J Natl Med Assoc 1984;76:157-61.

2. Fassler PR, Swiontkowski MF, Kilroy AW, Routt ML Jr. Injury of the sciatic nerve associated with acetabular fracture. J Bone Joint Surg [Am] 1993;75-A:1157-66

3. Hyslop GH. Injuries to the deep and superficial peroneal nerves complicating ankle sprain. Am J Surg 1941;51:436-8.

4. Lippin Y, Shvoron A, Yaffe B, Zwas ST, Tsur H. Postburn peroneal nerve palsy: a report of two consecutive cases. Burns 1993:9:246-8.

5. McMahon MS, Craig SM. Interfasicular reconstruction of the peroneal nerve after knee ligament injury. Ann Plast Surg 1994;32:642-4.

6. Nobel W. Peroneal palsy due to hematoma in the common peroneal nerve sheath after distal torsional fractures and inversion ankle sprains. J Bone Joint Surg [Am] 1966:48-A:1484-95

7. Sorrell DA, Hinterbuchner C, Green RF, Kalisky Z. Traumatic common peroneal nerve palsy: a retrospective study. Arch Phys Med Rehabil 1976;57:361-5.

8. Berry H, Richardson PM. Common peroneal nerve palsy: a clinical and electrophysiological review. J Neurol Neurosurg Psychiatry 1976;39:1162-71.

9. Burkhart FL, Daly JW. Sciatic and peroneal nerve injury: a complication of vaginal operations. Obstet Gynecol 1966;28:99-102

10. Curley P, Eyres K, Brezinova V, et al. Common peroneal nerve dysfunction after high tibial osteotomy. J Bone Joint Surg [Br] 1990;72-B:405-8.

11. Garland DE, Hughston JC. Peroneal nerve paralysis: a complication of extensor reconstruction of the knee. Clin Orthop 1979;140:169-71.

12. Miller DB Jr. Arthroscopic meniscus repair. Am J Sports Med 1988;16:315-20.

13. Rose HA, Hood RW, Otis JC, Ranawat CS, Insall JN. Peroneal-nerve palsy following total knee arthroplasty: a review of the Hospital for Special Surgery experience. J Bone Joint Surg [Am] 1982;64-A:347-51.

14. Slawski DP, Schoenecker PL, Rich MM. Peroneal nerve injury as a complication of pediatric tibial osteotomies: a review of 255 osteotomies. J Pediatr Orthop 1994; 14:166-72.

15. Weiss AP, Schenck RC Jr, Sponseller PD, Thompson JD. Peroneal nerve palsy after early cast application for femoral fractures in children. J Pediatr Orthop 1992;12: $25-8$ 
16. Kamada N, Nishioka T, Odu T, Shirotani H. Complications of the intraaortic balIoon pumping after open heart surgery. Japan J Thoracic Surgery 1993;46:858-61 (in Japanese).

17. Kantrowitz A, Wasfie T, Freed PS, et al. Intraaortic balloon pumping 1967 through 1982: analysis of complications in 733 patients. Am J Cardiol 1986;57:976-83.

18. Lederman RJ, Breuer AC, Hanson MR, et al. Peripheral nervous system complications of coronary artery bypass graft surgery. Ann Neurol 1982;12:297-301.

19. Clawson DK, Seddon HJ. The late consequences of sciatic nerve injury. J Bone Joint Surg [Br] 1960;42-B:213-25.

20. Ferguson FR, Liversedge LA. Ischaemic lateral popliteal nerve palsy. Br Med J 1954;488:333-5.

21. Mufson I. Diagnosis and treatment of neural complications of peripheral arterial obliterative disease. Angiology 1952;3:392-6.

22. Sunderland S. Blood supply of the sciatic nerve and its popliteal divisions, in man. Arch Neurol Psychiatr 1945;54:283-9.

23. Sunderland $\mathbf{S}$. The relative susceptibility to injury of the medial and lateral popliteal divisions of the sciatic nerve. Br J Surg 1953;167:300-2.

24. McManis PG, Low PA, Lagerlund TD. Microenvironment of nerve: blood flow and ischemia. In: Dyck PJ, Thomas PK, eds. Peripheral neuropathy. Vol. 1, Third ed. Philadelphia: W.B. Saunders Co, 1993:453-73.
25. Lundborg G, Brånemark P-I. Microvascular structure and function of peripheral nerves: vital microscopic studies of the tibial nerve in the rabbit. Adv Microcirc 1968; 1:66-88.

26. Flanigan DC, Cassell MD, Saltzman CL. Vascular supply of nerves in the tarsal tunnel. Foot Ankle Int 1997;18:288-92.

27. Bell MA, Weddell AGM. A descriptive study of the blood vessels of the sciatic nerve in the rat, man, and other mammals. Brain 1984;107:871-98.

28. Bell MA, Weddell AG. A morphometric study of intrafasicular vessels of mammalian sciatic nerve. Muscle Nerve 1984;7:524-34.

29. Tonkoff W. Die arterien der intervertebralganglien und der cerebrospinalnerven des menschen. Int Monatschr F Anat U Physiol 1898;15:353-401 (in German).

30. Hyrtl J. Ueber normale und abnorme verhaltnisse der schlagadern des unterschenkels. Wien: Buchandler der Kaiserlichen AK academie der Wissenschaften. Mathem naturw 1864:203-51 (in German).

31. Senior HD. The development of the arteries of the human lower extremity. Am J Anat 1919;25:55-95.

32. Senior HD. The development of the human femoral artery: a correction. Anat Rec 1920;17:271-9.

33. Mont M, Dellon AL, Chen F, et al. The operative treatment of peroneal nerve palsy. J Bone Joint Surg [Am] 1996;78-A:863-9. 